\title{
Féeries
}

Études sur le conte merveilleux, XVII $-\mathrm{XIX}{ }^{\mathrm{e}}$ siècle

$10 \mid 2013$

Conte et croyance

\section{Un Orient proche. Les volontés du Ciel dans Les Mille et Une Nuits}

A Close East: The Wills of Heaven in the Arabian Nights

Paul Pelckmans

\section{(2) OpenEdition}

12 Journals

Édition électronique

URL : http://journals.openedition.org/feeries/886

DOI : $10.4000 /$ feeries.886

ISSN : 1957-7753

Éditeur

UGA Éditions/Université Grenoble Alpes

\section{Édition imprimée}

Date de publication : 20 septembre 2013

Pagination : 137-152

ISBN : 978-2-84310-253-0

ISSN : $1766-2842$

\section{Référence électronique}

Paul Pelckmans, "Un Orient proche. Les volontés du Ciel dans Les Mille et Une Nuits », Féeries [En ligne], 10 | 2013, mis en ligne le 20 mars 2015, consulté le 08 septembre 2020. URL : http:// journals.openedition.org/feeries/886 ; DOI : https://doi.org/10.4000/feeries.886

\section{(c) Féeries}




\title{
UN ORIENT PROCHE. LES VOLONTÉS DU CIEL
}

\author{
DANS LES MILLE ET UNE NUITS
}

\author{
On ne peut pas espérer que les choses changent; c'est \\ un décret du Tout-Puissant qu'elles demeurent en cet \\ état. (t. II, p. $242^{\mathrm{I}}$ )
}

$L$

'IsLam Des Mille et Une Nuits d'Antoine Galland ne revêt pas vraiment une allure exotique. Le temps y est souvent rythmé par les cinq prières quotidiennes, avant lesquelles les personnages se lavent consciencieusement «les mains, le visage et les pieds» (t. I, p. 44); l'islam a aussi ses fêtes, comme "le baïram» (t. II, p. I9), et le culte a bien sûr lieu en des mosquées. Ces coutumes étrangères ne paraissent pourtant pas trop étranges puisqu'elles reproduisent à leur façon des pratiques dont les premiers lecteurs avaient pareillement l'habitude. Les voyageurs qui se rendent à La Mecque sont toujours des pèlerins, la liturgie égrène des fragments obligés de l'Alcoran comme elle fait ailleurs des pages de la Bible et l'aumône est de même une pratique très reconnaissable ${ }^{2}$. Tout cela affleure surtout à la faveur de notations incidentes et n'aboutit pas à un quelconque plaidoyer en faveur de l'islam, qui serait, dans le royaume du Très Chrétien, à peu près inimaginable; reste toujours que Galland ne dénigre jamais la religion de ses personnages, qui figure en somme une manière d'équivalent oriental de celle de ses lecteurs.

Il est difficile de jauger la portée précise d'une telle analogie, que Galland indique sans jamais hausser le ton, mais qui paraît à y réfléchir assez extraordinaire. Si elle suspend d'une part toute urgence missionnaire, il ne faut

I. Références (tome, page) aux Mille et Une Nuits. Contes arabes, traduction d'Antoine Galland, A. Chraïbi et J.-P. Sermain (éd.), Paris, GF Flammarion, 2004, 3 vol.

2. Notons au passage que les personnages d'Antoine Galland, sauf erreur, ne jeûnent jamais : l'islam des Nuits ignore curieusement le Ramadan, qui n'aurait pourtant pas surpris un public habitué au Carême. 
pas trop se hâter non plus d'y voir un pressentiment des Lumières. Les philosophes estimeront, comme on sait, que les ajouts des religions historiques à leur présumé commun dénominateur déiste sont foncièrement dérisoires et que mieux vaudrait supprimer au maximum ces excroissances inutiles. Les personnages des Nuits auraient plutôt raison de prolonger les pratiques établies de leurs religions respectives — et un des rares passages qui mettent en scène une confrontation d'obédiences différentes indique explicitement la leçon : "Ne prétendez pas obliger le monde à vivre à votre mode; vivez à la vôtre. " (t. I, p. I23) Ce qui ne signifie d'ailleurs pas que tel serait en ces parages le message fondamental des Nuits, où d'autres paragraphes dédaignent au nom de la foi musulmane la religion barbare des nègres de Sérendib ou l'idolâtrie des adorateurs du Feu. Galland ne se soucie pas de se montrer pour de bon conséquent - et c'est peutêtre surtout cela qui le distingue en profondeur des Lumières à venir : les philosophes se sentiront tenus de professer en ces parages des convictions dûment réfléchies et fort tranchées.

Antoine Galland nous ramène en ce sens dans un Ancien Régime où la religion établie fait figure d'évidence quotidienne et a priori respectable et où cette évidence profite cette fois, puisqu'on nous donne à lire des contes arabes, à une foi musulmane qui y assure un encadrement spirituel fort comparable à celui que les lecteurs connaissaient chez eux. L'islam des Nuits a même un profil d'autant plus «catholique» qu'on ne s'y contente pas de prier mais qu'on y choisit aussi très naturellement de commander des prières; nous avons bien affaire à une religion des æuvres, qui croit en la vertu de l'intercession payée de ses desservants. Un roi de Perse longtemps privé d'héritiers «faisait des aumônes immenses aux pauvres, de grandes largesses aux plus dévots de sa religion, et de nouvelles fondations toutes royales en leur faveur, afin d'obtenir par leurs prières ce qu'il souhaitait si ardemment» (t. II, p. 3II).

Ces largesses se répètent quand sa reine est enfin enceinte, puis quand elle accouche d'un garçon ${ }^{3}$. Tourmente agit de même pour retrouver son Ganem perdu :

Elle alla de mosquée en mosquée faire des largesses aux dévots de la religion musulmane, en implorant le secours de leurs prières pour l'accomplissement d'une affaire importante,

3. Voir t. II, p. 318 et 330. Voir aussi ce conseil du grand vizir du roi Shazaman : «Il n’y a que Dieu seul auquel on puisse recourir dans ces sortes de besoins. [...] Vous avez des sujets qui font une profession particulière de l'honorer, de le servir et de vivre durement pour l'amour de lui; mon avis serait que Votre Majesté leur fît des aumônes, et les exhortât de joindre leurs prières aux vôtres.» (t. II, p. I46) 
d'où dépendait, leur dit-elle, le repos de deux personnes. Elle employa toute la journée et ses mille pièces d'or à faire des aumônes dans les mosquées. (t. II, p. 4II)

On peut noter aussi, sur un tout autre plan, que Galland n'ignore évidemment pas - à la différence de beaucoup de ses lecteurs du Xxi ${ }^{\mathrm{e}}$ siècle que la religion de Mahomet hérite de la révélation biblique et vénère si l'on peut dire la même divinité. Les Nuits ne parlent jamais d'Allah, mais traduisent le vocable arabe par un "Dieu " très approprié, mais aussi très propre à amenuiser les distances. Quand un marchand, dès le tout premier conte de la série, s'avise de faire l'aumône "pour l'amour de Dieu» (t. I, p. 40), la distance se réduit un instant à zéro.

Il en va largement de même avec la bonne vingtaine de «Plût à Dieu que $^{4}$ » qui ponctuent à l'occasion les propos des personnages. Les assez nombreuses allusions des Nuits à certaine conduction divine du monde et à son impact inéluctable ne renvoient pas forcément à un fatalisme qui serait typiquement oriental. L'idée, certes, s'impose elle-même assez «fatalement» : le cliché d'un tel habitus oriental a la vie dure et Galland cherche de toute évidence à faire découvrir un ailleurs qui le passionne. Quand on voit en outre que les romanciers et conteurs contemporains qui situent leurs fictions dans un décor européen font rarement état d'une telle conduction céleste, il semble tentant de conclure qu'une référence qui, dans le monde du roman s'entend, n’apparaît guère qu'ici doit être nécessairement «orientale».

Cela n'est pourtant pas évident. Si les romanciers de la fin du XviI ${ }^{\mathrm{e}}$ siècle se réferent peu à la volonté divine, leur discrétion relève d'abord d'un silence respectueux, qui se distingue de celui des jansénistes parce qu'il n'implique, bien au contraire, aucune réserve mentale. Ils s'abstenaient de faire état de la volonté de Dieu au sujet de personnages et d'incidents inventés pour le seul plaisir parce que ce serait Lui manquer de respect. Le Second Commandement, qui proscrit le parjure, interdit aussi d'utiliser le nom de Dieu «en vain» (Exode, XX, 7); il semblait indécent de le compromettre parmi les frivolités de la fiction, où l'on ressuscitait à l'occasion les dieux de la mythologie — ou, dans L'Astrée, ceux des anciens Gaulois —, mais où l'on évitait de trop mentionner le vrai Dieu. L'islam des Nuits ressemble au catholicisme régnant, mais reste en même temps une fausse religion, qu'on pouvait convoquer de façon plus désinvolte. Antoine Galland ne s'en prive pas.

4. Voir entre autres t. I, p. 95, I94, 350 et 422; t. II, p. 79, 9I, IO4, I2I, I3I, I5I, I68, I77, 220, 266, 350, 392, 397, 493, 498; t. III, p. I3I. 
Ce qui ne signifie d'ailleurs pas qu'il voyait là une aubaine. On sait que l'auteur des premières Nuits françaises travaille à partir de sources qui ignorent pour leur part le tabou que nous venons d'évoquer et où les références religieuses se font souvent très insistantes. Son adaptation les réduit à une portion congrues, qui déborde certes ce qu'on trouve communément chez ses contemporains à lui, mais qui reste toujours très en-deçà de ce que proposaient les manuscrits originaux. On note de même que ces références se font nettement plus rares, même si elles n'y disparaissent bien sûr pas, dans ses derniers contes, soit en gros dans le tome III de notre édition de référence, où Galland, comme on sait, ne rédige plus à partir de manuscrits, mais de récits oraux et de notes d'écoute. Il cherche toujours à y prolonger la manière qui avait fait son succès et y réussit fort bien puisqu'Ali Baba et Aladdin resteront pour des générations de lecteurs les héros les plus populaires de son chef-d'œuvre; cela aussi donne à penser que la référence aux desseins du Ciel, pour voyante qu'elle soit tout au long des Nuits, n'y est décidément pas un enjeu central.

Galland fait écho, en mineur et sans s'y intéresser outre mesure, à certaine déférence insistante de ses originaux pour les volontés du Ciel. Tout se passe comme s'il escomptait là encore que ses lecteurs sauraient toujours s'y reconnaître ${ }^{6}$. Les Nuits se contenteraient en somme de transcrire un peu plus copieusement qu'à l'accoutumée des postures dont leur public s'étonnait aussi peu que les premiers auditeurs "arabes» puisqu'il les partageait toujours largement. C'est ces postures-là, qui nous sont devenues depuis fort étrangères, qu'il s'agira ici de cerner.

Les quelques centaines de notations qui les laissent entrevoir, et qu'Antoine Galland a dû écrire pour la plupart au courant de la plume et sans y réfléchir à chaque fois, ne dessinent pas un système vraiment cohérent. Les références à la volonté de Dieu, voire à sa Providence, pour être sans doute les causalités surnaturelles les plus souvent invoquées, voisinent avec des propos qui évoquent plutôt des forces impersonnelles telles la fortune, le

5. Voir au sujet de ces réductions, S. Larzul, Les Traductions françaises des Mille et Une Nuits. Étude des versions Galland, Trébutien et Mardrus, Paris, L'Harmattan, 1996, p. 78-86.

6. Georges May, dans son ouvrage pionnier, suggérait que tels thèmes pour nous d'office exotiques des Mille et Une Nuits "pouvaient bien être de moindres occasions de dépaysement pour le public d'alors que pour nous» (G. May, Les Mille et Une Nuits d'Antoine Galland, Paris, PUF, I986, p. 210). La présente étude voudrait en somme démontrer que cela vaut particulièrement pour les arrière-plans religieux des Nuits. Ou encore, en détournant cette fois une formule de Jean-Paul Sermain sur le style de Galland, qui cherche à rendre les aspects religieux de son Orient «aussi immédiats et communs que possible» (Les Mille et une nuits entre Orient et Occident, Paris, Desjonquères, 2009, p. 93) pour un public qui reste de toute évidence très majoritairement chrétien. 
sort ou la destinée. Il arrive aussi, très rarement il est vrai, qu'un personnage se sente «entraîné par [s] on étoile» (t. I, p. 274) ou "par la fatalité des astres» (t. III, p. 4I3 $3^{7}$ ). Ce syncrétisme doit tenir pour une part à la diversité des matériaux utilisés; elle n’explique pas tout puisque des vocables hétérogènes, et qui seraient à la limite logiquement incompatibles, affleurent à l'occasion dans la même phrase ou au sujet d'incidents tout à fait comparables. Voici d'abord le naufrage du "Troisième Calender» :

Tous mes gens furent noyés; mais Dieu eut pitié de moi, et permit que je me sauvasse en me saisissant d'une planche qui fut poussée par le vent droit au pied de la montagne. Je ne me fis pas le moindre mal, mon bonheur m'ayant fait aborder à un endroit où il y avait des degrés pour monter au sommet. (t. I, p. I77)

Sindbad le marin cumule sept naufrages; lors du premier, il doit son salut à "quelques racines d'arbres que la fortune semblait avoir conservées en cet endroit» (t. I, p. 234) et qui lui permettent de grimper sur un récif au pied duquel il se serait autrement noyé. Après le troisième, il finit par retrouver très vite le capitaine qui l'avait abandonné par erreur et qui est tout heureux de le revoir vivant : «Dieu soit loué! s'écria-t-il en m’embrassant; je suis ravi que la fortune ait réparé ma faute.» (t. I, p. 254)

Zobéide, pour sa part, ne fait pas naufrage, mais est jetée à la mer par des sœurs envieuses. Elle réussit à nager quelque temps, puis finit, "par bonheur ou plutôt par miracle» par «trouv[er] fond» (t. I, p. 2I4) près d'une île déserte.

Ces explications invoquées plus ou moins au hasard relèvent d'une pensée toute faite, qu'Antoine Galland prolonge sans vraiment l'interroger. Chaque vocable a bien sûr sa saveur propre. La fortune est «inconstante» (t. I, p. 95) et portée aux changements à vue; la destinée impose plutôt un sort immuable et est souvent invoquée au sujet de la mort ( «la destinée de l'homme est de mourir", t. I, p. 49), dont elle fournit le cas échéant une cause suffisante : «son heure était venue, et c'est la cause unique de sa mort» (t. III, p. 484). La volonté de Dieu est évoquée surtout — mais pas exclusivement, nous y reviendrons - au sujet d'événements favorables, qui attestent certaine sollicitude du Créateur pour ses créatures. On trouve même à l'occasion des phrases où la fortune et le Ciel semblent se partager les rôles. Un vieil homme recommande à un prince naufragé, qui tient à son incognito, de se faire jardinier :

7. Voir aussi la promesse cocasse du Barbier importun : «Je veux [...] m'attacher à vous, vous prendre sous ma protection et vous garantir de tous les malheurs dont les astres pourront vous menacer.» (t. I, p. 427) 
Par ce moyen, vous vous mettrez en état d'attendre que le Ciel vous soit favorable, et qu'il dissipe le nuage de mauvaise fortune qui traverse le bonheur de votre vie. (t. I, p. I49)

La question de savoir pourquoi le Ciel diffère de «dissiper le nuage» ne se pose pas... Il en va de même lors d'un dénouement heureux inattendu, dont les témoins «ne pouvaient retenir leurs larmes, ni se lasser d'admirer la Providence, qui rassemblait chez eux quatre personnes que la fortune avait si cruellement séparées" (t. II, p. 4I8).

L'idée ne vient à personne qu'il aurait été plus admirable encore de leur éviter cette cruelle séparation. Il est vrai que pareille prévenance aurait supprimé du coup le conte, qui n'existe que d'aboutir seulement à un dénouement heureux.

Les phrasés tendancieux qui réservent le beau rôle à Dieu restent à tout prendre assez rares. Les Mille et Une Nuits ne critiquent pas la Providence, mais ne cherchent pas non plus à la défendre. Les personnages ont souvent à se louer du Ciel et le remercient volontiers; il n'y a là aucun parti pris apologétique, mais un simple effet de genre puisque les contes, par définition, se terminent généralement bien et sont si peu tourmentés par le problème du mal et du malheur qu'ils admirent aussi que ce bonheur final soit généralement atteint après bien des tribulations :

Dieu soit loué de toutes choses, et particulièrement de cette aventure merveilleuse, qui montre si bien sa puissance! (t. I, p. 330)

Le calife Haroun-al-Raschid félicite de même tel de ses conteurs parce que «les voies dont il a plu à la Providence de (le) gratifier de ses dons (ont dû) être extraordinaires» (t. III, p. I49); la formule précède le récit de l'intéressé et définit donc un horizon d'attente, que l'histoire qui suit ne dément d'ailleurs pas plus que toutes les autres.

Cogia Hassan Alhaball, qui en est le protagoniste, ne remercie pas seulement le Ciel pour sa richesse finale. Il aura été d'abord, avec sa famille, un pauvre content de son sort :

Il nous suffit que nous soyons contents du peu que Dieu nous donne, et qu'il nous ôte la connaissance et le désir de ce qui nous manque. (t. III, p. 152)

Un voisin riche se livre alors à une curieuse expérience en lui offrant "une bourse de deux cents pièces d'or" pour voir s'il saura en faire "un bon usage» (t. III, p. I53); l'essai tourne court presque aussitôt quand un milan enlève le turban où Cogia avait caché sa bourse. Il se retrouve donc pauvre comme avant, se voit en outre soupçonné d'avoir misérablement gaspillé sa petite fortune, mais ne se révolte pas : 
Dieu, disais-je, a voulu m'éprouver en me donnant du bien dans le temps que je m’y attendais le moins : il me l'a ôté presque dans le même temps, parce qu'il lui a plu ainsi et qu'il était à lui. Qu'il en soit loué, comme je l'avais loué jusqu'alors des bienfaits dont il m’avait favorisé, tels qu'il lui avait plu aussi! Je me soumets à sa volonté. (t. III, p. I56)

Le voisin riche lui donne pourtant sa seconde chance, qui finit tout aussi piteusement : Cogia cache sa nouvelle fortune "au fond d'un grand vase de terre plein de son» (t. III, p. I58), que sa femme vend inévitablement le même jour à un marchand ambulant. Cogia ne se révolte toujours pas :

Seigneur, dis-je en m’adressant à [mon voisin riche], il n'a pas plu à Dieu que votre libéralité servît à m’enrichir, par un de ses secrets impénétrables que nous ne devons pas approfondir. Il me veut pauvre, et non pas riche. (t. III, p. I62)

Quand on lui donne alors, en riant, un simple morceau de plomb, ce don dérisoire l'enrichit définitivement : Cogia le passe le lendemain à un voisin pêcheur qui doit raccommoder ses filets et qui lui promet en guise de paiement le produit de sa première pêche; cette pêche lui vaut un seul gros poisson («Dieu ne m'a envoyé pour vous que celui-ci», t. III, p. I65), dont le ventre se trouve, par la suite, contenir un gros diamant. Quelques jours plus tard, d'autres hasards heureux, où Cogia reconnaît pareillement le doigt de $\mathrm{Dieu}^{8}$, prouvent à tout le monde que ses récits sur le milan voleur et sur le vase de terre malheureusement vendu avaient été véridiques.

L'infinie patience de Cogia rejoint celle du saint homme Job, dont l'histoire peut fort bien répercuter un lointain écho. L'idée qu'il convient d'agréer aussi les malheurs envoyés par le Ciel est de toute manière une leçon hautement traditionnelle. Les Mille et Une Nuits, en dépit de leur note génologique souriante, la reprennent à y regarder de près plus souvent qu'on ne dirait — à tel point qu'on pourrait dire que cette injonction pour nous assez austère mais qui pouvait se profiler encore, du temps d'Antoine Galland, comme une manière de truisme, forme en quelque sorte leur basse continue. Les Nuits d'une œuvre s'attardent bien sûr plus volontiers aux «voies toutes merveilleuses» (t. III, p. I79) où leurs personnages finissent à chaque fois par trouver leur bonheur, mais encadrent aussi ces belles luxuriances dans une soumission quasi instinctive au cours du monde comme il va et à la décision divine qui en dicterait les aléas.

8. Voir «Plein de joie, je dis à mes bienfaiteurs : Seigneurs, Dieu ne veut pas que vous vous sépariez d'avec moi que vous ne soyez pleinement convaincus de la vérité dont je n’ai cessé de vous assurer.» (t. III, p. I78) 
Les théologiens des grandes religions auront dépensé des trésors d'ingéniosité pour décrire les voies de la Providence et lui attribuer des modalités parfois fort subtiles qui devaient la rendre compatible avec la bonté et/ou la justice du Ciel ou avec la liberté humaine. Les Mille et Une Nuits n'ont guère à voir avec ces exercices de haute voltige ${ }^{9}$; Galland a plus de chances de nous édifier (c'est le cas de le dire) sur les tenants et les aboutissants d'une religiosité plus vernaculaire ${ }^{\mathrm{IO}}$, faite des croyances et des attitudes communément partagées par la masse des croyants ordinaires.

La Providence est un des dogmes centraux de cette religion populaire ${ }^{\text {II, }}$ qui admet sans trop de questions qu'un Dieu sage et tout-puissant dirige les choses pour le mieux. Cela ne signifie toujours pas, pour les chrétiens traditionnels de l'Ancien Régime, qu'Il serait censé s'inquiéter constamment du bien-être quotidien de chacune de ses créatures. Même s'il existe bien sûr des anecdotes édifiantes qui abondent dans ce sens - et même des proverbes qui le généralisent (à brebis tondue Dieu ménage le vent) —, l'Âge Classique est trop peu porté à s'intéresser au particulier pour ne pas admettre que le Ciel se contente raisonnablement d'une conduction globale du monde. Au XvII ${ }^{\mathrm{e}}$ comme au début du XvIII ${ }^{\mathrm{e}}$ siècles, le Bon Dieu n'entre pas encore dans le détail et ne doit pas le faire : il lui suffit d'établir un ordre commun qui comporte sans doute pas mal de désagréments et les répartit même de manière fort inégale, mais dont tous les intéressés n’ont finalement qu'à s'accommoder.

La prédication chrétienne ajoutait volontiers qu'on pouvait faire son salut dans n'importe quelle condition et que la différence des sorts terrestres ne faisait guère le poids à côté des récompenses et des peines éternelles également promises à tout le monde. Les Nuits parlent très incidemment ${ }^{12} \mathrm{de}$ l'au-delà et ne devaient pas en faire plus puisqu'on y devient généralement

9. Il est tout au plus amusant de penser que le chef-d'œuvre d'Antoine Galland paraît au moment même où Leibniz élaborait de son côté la théodicée la plus subtile de toute l'histoire de la philosophie.

Io. On pourrait rappeler aussi la distinction, chère à Jean Delumeau, entre le prescrit et le vécu. Voir par exemple sa leçon d'ouverture au Collège de France qui porte ce titre (à lire entre autres dans J. Delumeau, Le Christianisme va-t-il mourir?, Paris, Hachette, I977, p. I79-2I4).

II. Une bonne histoire des idées populaires sur la Providence reste largement à écrire. Les paragraphes qui suivent se basent sur quelques coups de sonde, dont on retrouvera l'essentiel dans mon Problème de l'incroyance au XVIII siècle, Laval, Presses de l'Université, 20IO, p. 25-70.

I2. Je n'ai noté pour ma part (liste sans doute incomplète) qu'une dizaine d'allusions; voir t. I, p. I4I, I96, 2I8, 293, 296, 373; t. II, p. 77, 269, 307, 382, 408. Une étude exhaustive de l'eschatologie des Nuits devrait bien sûr se demander aussi si telles escales de ses personnages voyageurs ne les amènent pas dans certain au-delà — ou du moins en des paysages qui prolongent d'anciennes scénographies eschatologiques. 
heureux dès l'ici-bas; les croyants en chair et en os de la vie réelle ne pouvaient bien entendu compter sur des issues si invariablement favorables! Toujours est-il que, même chez eux, l'argument eschatologique ne faisait sans doute qu'une considération d'appoint, qui justifiait (ou «rationalisait») à sa façon une déférence fondamentale qui est une posture de base de toutes sociétés traditionnelles. Le dogme de la Providence, dans ce sens, n'appelait pas à s'émouvoir de l'infinie sollicitude d'un Dieu affectueux, ni même à proprement parler à admirer sa justice; il conviait surtout à obéir sans murmure à une Toute-Puissance qui dirigeait impérieusement le cours du monde ${ }^{13}$.

De telles attitudes nous sont devenues profondément étrangères. Notre modernité individualiste est convaincue de l'importance primordiale de chaque sujet personnel et ne saurait admettre que personne puisse être tenu à s'aligner sans récriminations, et à la place qui lui est échue, sur le monde comme il va. L'Âge Classique participe encore d'une «culture de la soumission ${ }^{14} »$ sans âge où cet impératif paraissait au contraire évident; ce serait même surtout par là qu'il reste à sa façon très proche du monde des Nuits. Le respect inconditionnel de la Providence consonait avec ce régime holiste et le parachevait en consacrant un ordre global qui prévalait depuis toujours sur toutes prédilections et initiatives particulières.

Les Mille et Une Nuits permettent de retrouver quelques linéaments de ces postures révolues. Il est vrai que les conteurs ne précisent jamais que les tribulations qui précèdent leurs dénouements heureux correspondraient elles aussi à une volonté du Ciel, qu'il conviendrait donc de respecter en tant que telle. L'argument serait théologiquement imparable, mais imposerait un peu directement l'image d'une divinité qui soufflerait selon ses caprices le froid et le chaud. Il s'ébauche une seule fois - et s'incline alors devant une autorité terrestre, d'ailleurs à sa façon de droit divin. Le Dormeur Éveillé Abou Hasan se console aussitôt d'une série de péripéties très éprouvantes quand il comprend qu'il s'agissait d'une plaisanterie savamment montée de Haroun-al Raschid :

Commandeur des croyants, [...] quelque grands que soient les maux que j'ai soufferts, ils sont effacés de ma mémoire du moment que j'apprends qu'ils me sont venus de la part de mon souverain seigneur et maître. (t. II, p. 476)

I3. Voir, parmi mille, cette citation de Bossuet : «Il nous importe peu, chrétiens, de connaitre par quelle sagesse nous sommes régis, si nous n'apprenons aussi à nous conformer à l'ordre de ses conseils. S'il y a de l'art à bien gouverner, il y en a aussi à bien obéir." (Sermon sur la Providence, dans C. Cagnat-Deboeuf (éd.), Carême du Louvre, Paris, Gallimard, 20oI, p. II3.)

I4. Terme de M. Laget, Naissances. L'accouchement avant l'âge de la clinique, Paris, Seuil, I982, p. 125 . 
Le calife, pour autant qu'on voie, a monté son stratagème pour son seul amusement; le conte nous ramène à une époque où ce bon plaisir suprême valait une raison suffisante.

On sait que Haroun-al Raschid se sera offert en l'occurrence « un divertissement tout singulier» (t. II, p. 435), où Abou Hassan finit par ne plus savoir quand il rêve et quand il est éveillé après qu'on l'a transporté tout endormi de son propre lit dans celui du calife, puis ramené chez lui dès la nuit suivante. Le lecteur moderne ne peut manquer de se souvenir de La Vie est un songe ${ }^{15}$. Du point de vue qui nous intéresse ici, il importe surtout de noter que le trouble identitaire qui fait le fond de l'anecdote n'amène jamais Hassan à dénoncer sa soumission ${ }^{16}$. Se réveillant dans le lit du calife, il renonce à se demander s'il rêve ou non : "Quoiqu'il en soit, je me remets à Dieu sur ce qui en est.» (t. II, p. 446) Il n’y a peut-être pas, à ce moment, un très grand mérite parce que la situation où il se retrouve inopinément est tout sauf désagréable. Le lendemain, le réveil est moins lénifiant: Abou ramené chez lui se croit toujours calife, maltraite sa mère qui essaie de le raisonner et se voit conduit, parmi les huées et les outrages de ses voisins, à l'hôpital des fous furieux :

À tous ces mauvais traitements : «Il n’y a, disait-il, de grandeur et de force qu'en Dieu très haut et tout-puissant. On veut que je sois fou quoique je sois dans mon bon sens; je souffre cette injure et toutes ces indignités pour l'amour de Dieu.» (t. II, p. 458)

Quand sa détention se prolonge et qu'aucun des dignitaires qu'il a «vu à [s] es pieds» (t. II, p. 459) ne vient l'en tirer, il finit par se dire que son califat d'un jour doit être une chimère, même s'il se souvient toujours qu'il avait aussi pu remarquer le lendemain que certains ordres qu'il avait donnés avaient été bel et bien exécutés. Il ne lui reste donc qu’à s’accommoder de ces mystères insolubles :

Cela m'arrête et je n'y comprends rien. Mais combien d'autres choses y a-t-il que je ne comprends pas et que je ne comprendrai jamais! Je m'en remets donc entre les mains de Dieu qui sait et qui connaît tout. (t. II, p. 46o)

15. Les lecteurs contemporains ont du être moins nombreux à faire le rapprochement : la première traduction française de la pièce, due à Thomas-Simon Gueulette, ne paraîtra qu'en I723. Je rappelle au passage que Calderón a pu se souvenir de son côté d'une légende musulmane — même si Lucien Dupuis va sans doute un peu vite en affirmant tout uniment, dans sa notice de la Pléiade, que «la fable du dormeur transporté sur le trône (serait) tirée d'un conte des Mille et Une Nuits» (Théâtre Espagnol du XVII Siècle II, Paris, Gallimard, 1999, p. 1787). C'est oublier — après bien d'autres — que les Nuits n'existent, dans toutes les acceptions de la formule, qu'à partir du chef-d'œuvre d'Antoine Galland...

I6. On se souvient que Sigismond, chez Calderón, finit par décider qu'il se conduira, que sa royauté soit réelle ou non, en bon prince et se dit sûr de gagner ainsi le Ciel. Dans son cas aussi, le doute sur soi ne fait vaciller ni l'évidence des devoirs ni les certitudes religieuses.

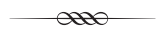


Quelques jours plus tard, il se réveille de nouveau dans le lit du calife, où il essaie cette fois de se garder de son mieux d'une illusion dont il sait désormais qu'elle risque de tourner très mal et qu'il prend donc pour une machination de Satan. On est donc peu surpris de le voir appeler Dieu à l'aide contre cette "tentation» (t. II, p. 470); son califat, qui ne dure cette fois qu'une bonne heure, lui laisse le temps pour trois prières jaculatoires, où il répète avec de légères variantes qu'il se remet "entre les mains" (t. II, p. 470 et 472) de la Providence — au hasard de tout ce qu'une telle soumission risque de devoir agréer dans une situation aussi douteuse. Après quoi le vrai calife survient enfin pour le tirer de ses perplexités; Abou aurait eu quelque raison de se plaindre, mais reçoit ses éclaircissements avec la déférence qu'on sait.

Qui doute de soi-même devrait, dirions-nous, en venir rapidement à douter de tout : l'individu moderne se croit au centre de son monde et l'entraîne donc dans sa chute quand il vient à perdre ses repères. Le désarroi savamment fomenté d'Abou Hassan semble moins contagieux et ne fragilise jamais que lui-même. Il reste même assez limité : le Dormeur Éveillé ne sait plus qui il est, mais finit aussi bien par se résigner à l'ignorer comme il ignorera à jamais tant d' "autres choses» : l'alignement est d'une belle impartialité et prouve qu'il n'en est pas encore à revendiquer une quelconque priorité existentielle pour des affres qui nous sembleraient intolérables. Il lui suffit de savoir que son devoir essentiel sera toujours de se plier à tout ce qui lui arrive.

Il n'y faut d'habitude pas tant de souplesse. L'univers de la soumission est en règle générale un monde hiérarchisé, où les décalages entre les conditions sont unanimement reconnus et où chacun reste à sa place et est même tenu d'y rester. Les inégalités sociales paraissant quasiment fondées en nature, il serait à la fois incongru et indécent de prétendre sortir d'un état auquel on appartient depuis sa naissance et qui se confond en pratique pour chaque être humain avec son identité personnelle.

Les Nuits ne prêchent pas plus ce respect des conditions qu'elles ne plaident la cause de la Providence. On y voit des princes qui se font aidejardinier ou un fils de calife qui gagne sa vie comme pâtissier; il suffit pour cela qu'un naufrage ou quelque malin génie les amène dans quelque pays fort lointain d'où ils ne peuvent pas repartir de sitôt et où il faut bien subsister comme on peut. Eux aussi respectent alors la règle puisque ces rescapés prennent à chaque fois assez l'esprit de leur nouvel état pour s'y résigner, parfois des années durant, sans récriminer. Le commun des mortels n'est généralement pas exposé à ces changements à vue : les contes multiplient les départs fabuleux, mais présupposent un monde où chacun reste plutôt sur place et y reste aussi à sa place. 
Cette évidence première ne vient guère à s'exprimer en direct. On en trouve au moins un assez curieux écho dans les quelque six épisodes des Nuits où des personnages métamorphosés en animaux par tel ou tel maléfice se voient ramenés par une nouvelle conjuration à leur forme humaine. Ces métamorphoses, qu'on imagine devoir être un des universaux de l'imaginaire merveilleux, engageraient à première vue l'intuition d'un monde infiniment ductile où aucune identité ne serait durablement acquise; elles se combinent toujours dans les Nuits avec un foncier essentialisme. Les sortilèges positifs s'y efforcent en effet de restaurer une forme originelle qui serait la vérité vraie, et voulue par Dieu, de l'individu concerné et récusent aussi tout autre changement :

Ô veau! dit-elle, si tu as été créé par le tout-puissant et souverain maître du monde tel que tu parais en ce moment, demeure sous cette forme; mais, si tu es homme et que tu sois changé en veau par enchantement, reprends ta figure naturelle par la permission du souverain Créateur. En achevant ces mots, elle jeta l'eau sur lui, et à l'instant il reprit sa première forme. (t. I, p. 56; italiques de Galland)

Si tu es né chien, demeure chien; mais si tu es né homme, reprends la forme d'homme par la vertu de cette eau. À l'instant, l'enchantement fut rompu : je perdis la figure de chien et je me vis homme comme auparavant. (t. I, p. 56; italiques de Galland)

Une méchante sorcière qu'on amène à annuler son propre maléfice, prononce d'abord pour sa principale victime une formule très comparable ${ }^{17}$. Elle désenchante ensuite toute une ville qu'elle avait transformée en lac et où les habitants retrouvent des différences restées immuables :

La ville reparut à l'heure même. Les poissons redevinrent hommes, femmes ou enfants; mahométans, chrétiens, persans ou juifs, gens libres ou esclaves, chacun reprit sa forme naturelle. Les maisons et les boutiques furent bientôt remplies de leurs habitants qui y trouvèrent toutes choses dans la même situation et dans le même ordre où elles étaient avant l'enchantement. (t. I, p. IIo; je souligne)

Pareilles métamorphoses illustrent à leur manière l'essentielle stabilité — et la tout aussi essentielle hétérogénéité — d'un monde où l'on ne doit jamais retrouver pour finir que sa propre forme.

Une troisième magicienne précise en toutes lettres qu'elle prononce sa formule libératrice "au nom du Créateur du ciel et de la terre, qui ressuscite les morts et maintient l'univers dans son état» (t. II, p. 353; italiques de Galland). Il faudrait un esprit chagrin pour objecter qu'un monde où les

17. Voir : «Si le Créateur de toutes choses t’a formé tel que tu es présentement, ou s'il est en colère contre toi, ne change pas; mais, si tu n'es dans cet état que par la vertu de mon enchantement, reprends ta forme naturelle.» (t. I, p. IO8) 
morts ressusciteraient ne resterait pas précisément dans son état; la formule associe deux phrases toutes faites et sous-entend bien sûr, puisqu'on ne le sait que trop, que les résurrections miraculeuses restent rares, mais qu' elles illustrent une infinie Toute-Puissance qui garantit dans ses œuvres coutumières certaine fondamentale continuité du monde - et dont on espère ici un autre prodige puisqu'il s'agit en l'occurrence de rendre un oiseau à sa forme humaine originelle ${ }^{18}$.

Le Ciel décide de la diversité des espèces comme de celle des conditions. Il décide aussi de la durée de la vie d'un chacun ${ }^{19}$. Les personnages des Nuits se montrent là encore exemplairement soumis. Les contes multiplient — et apprécient — les sauvetages de la toute dernière minute et les amènent par les biais les plus imprévisibles; ils racontent du coup plus d'une situation apparemment sans issue, où les intéressés se résignent alors sans trop murmurer à une mort qui reste, pour reprendre le terme bien connu de Philippe Ariès, très apprivoisée. Dès le tout premier conte de Shéhérazade, le marchand qui a obtenu un sursis du génie qui l'avait condamné à mort, se montre fidèle au rendez-vous qu'il a juré de respecter; comme il a pris "Dieu à témoin» (t. I, p. 48), il ne lui reste, le moment venu, qu’à prendre congé de sa famille :

Mes enfants, leur dit-il, j'obéis à l'ordre de Dieu en me séparant de vous. Imitez-moi : soumettez-vous courageusement à cette nécessité, et songez que la destinée de l'homme est de mourir. (t. I, p. 49)

Un pécheur qui encourt la colère d'un autre génie se passe même de tout sursis :

Puisque je ne saurais éviter la mort, dit-il au génie, je me soumets donc à la volonté de Dieu. (t. I, p. 69)

L'un et l'autre, comme on sait, finiront par avoir la vie sauve. Aladdin, quand il se trouve d'abord enfermé dans la fameuse caverne aux trésors, se voit même sauvé à la faveur de sa pieuse résignation :

I8. Il est à peine besoin de souligner que Galland, même s'il met à chaque fois des italiques (voir aussi t. I, p. I7I), ne transcrit pas ces formules pour édifier son public, mais pour le seul plaisir de circonstancier ses épisodes merveilleux. Un de nos six épisodes choisit d'ailleurs de ne pas entrer dans ce détail et indique simplement que la fée qui y est de service "prononça [...] des paroles que personne n'entendit» (t. I, p. 227).

19. Notons au passage que les fées, que nous avons vues très performantes dans le domaine des métamorphoses, ne se risquent jamais à ressusciter des morts. Pari Banou se flatte de pouvoir remédier à tous les chagrins de son époux très aimé - et ajoute une seule exception : «il faudrait que [la cause de votre peine] fût bien extraordinaire si elle était hors de mon pouvoir, à moins que ce ne fût la mort du sultan votre père; en ce cas-là, avec ce que je tâcherais d'y contribuer de mon côté, le temps vous en apporterait la consolation» (t. III, p. 3I4). 
Aladdin demeura deux jours [...] sans manger et sans boire; le troisième jour enfin, en regardant la mort inévitable, il éleva les mains en les joignant, et, avec une résignation entière à la volonté de Dieu, il s'écria : "Il n'y a de force et de puissance qu'en Dieu le haut, le grand!»

Dans cette action de mains jointes, il frotte sans y penser l'anneau que le magicien africain lui avait mis au doigt et dont il ne connaissait pas encore la vertu. (t. III, p. 22)

C'était le geste qu'il fallait accomplir pour convoquer le génie de l'anneau, qui le tire "aussitôt» (t. III, p. 22) de son mauvais pas. On notera que ce sauvetage ne récompense pas la dévotion d'Aladdin; il se trouve seulement qu'elle l'amène, par hasard, au geste idoine. Un conteur d'esprit plus apologétique aurait pu sauter sur l'occasion pour insinuer que la dévotion est toujours (ou quelquefois) payante; notre conte nous ramène dans un monde où la mort n'a rien de scandaleux et où Dieu n'est donc pas tenu d'en sauver ses fidèles.

La même résignation revient au sujet de telle mort effective :

[Noureddin Ali] fut attaqué tout à coup d'une maladie dont la violence fut telle qu'il sentit fort bien qu'il n'était pas éloigné du dernier de ses jours. Aussi ne se flatta-t-il pas, et il se disposa d'abord à mourir en bon musulman. (t. I, p. 3IO)

Elle revient même au sujet de quelques décès inventés de toutes pièces. Quand les princes Amgiad et Assad sont condamnés sur la foi d'une terrible calomnie, leur bourreau apitoyé les laisse s'échapper et ne tarit pas, par la suite, sur leur «constance admirable» et leur «soumission inconcevable à leur arrêt de mort» (t. II, p. 223); le roi trompé qui avait prononcé l'arrêt est d'autant plus porté à y réfléchir de plus près et ne tarde pas à découvrir leur innocence! Le magicien qui se fait passer pour l'oncle d'Aladdin s'accrédite de même auprès de sa prétendue parentèle en trouvant d'emblée le ton voulu au sujet de la mort de celui qui serait dès lors son frère :

[Aladdin] a pu vous dire de quelle manière j'ai reçu la triste nouvelle qu'il n'était plus au monde; mais il faut louer Dieu de toutes choses. (t. III, p. II)

Ganem et son épouse Nouzhatoul-Aouadat s'amusent pour leur part à semer une confusion inextricable à la cour de Haroun-al Raschid en racontant simultanément chacun de son côté, la mort subite de l'autre conjoint. La référence aux volontés du Ciel s'impose alors d'autant plus qu'elle est la meilleure explication de ces fins mensongères que rien, et pour cause, ne laissait prévoir. Ganem termine ainsi son récit du coup qui serait venu le frapper :

Elle devait mourir, et Dieu n'a pas voulu me laisser jouir plus longtemps d'un bonheur que je tenais des bontés de Votre Majesté et de Zobéide, sa chère épouse. (t. II, p. 487) 
Comme Nouzhatoul-Aouadat faisait partie de la suite de la sultane, le sultan console aussi son épouse :

Nous sommes tous mortels, et nous devons rendre à Dieu la vie qu'il nous a donnée quand il nous la demande. (t. II, p. 488)

Zobéide n'a entendu parler de son côté que de la mort de Ganem et est évidemment très étonnée. On décide donc d'envoyer le chef des eunuques visiter le ménage endeuillé; comme les deux complices préfèrent à ce moment le faire croire à la mort de l'épouse et ont le temps d'arranger une mise en scène, Nouzhatoul-Aouadat fait la morte sous une pièce de brocart. L'eunuque lui fait ses adieux :

Il n'y a pas d'autre Dieu que Dieu, dit-il avec un soupir profond. Nous devons nous soumettre tous à sa volonté, et toute créature doit retourner à lui. NouzhatoulAouadat, ma bonne sœur, ajouta-t-il en soupirant, ton destin a été de peu de durée! Dieu te fasse miséricorde! (t. II, p. 492)

Le souhait final rendait, au début du XviıI ${ }^{\mathrm{e}}$ siècle, un son très familier ${ }^{20}$; aussi la nourrice de la sultane le reprend-elle quand on l'envoie derechef aux nouvelles à un moment où Ganem fait à son tour le mort ${ }^{21}$.

Cette plaisanterie prolongée - et pour nous plutôt macabre - finit, quand les complices dévoilent enfin leurs batteries, par faire s'esclaffer tout le monde. Cela aussi atteste à sa façon la foncière acceptation de Thanatos qui est un des traits les plus caractéristiques des cultures de la soumission. On peut en dire autant, tout bien considéré, des Mille et Une Nuits elles-mêmes, où le voisinage constant avec une mort toujours imminente n'empêche jamais Shéhérazade de réciter ses «beaux contes» de la façon la plus enjouée.

Antoine Galland, on aura pu s'en convaincre, se distingue entre autres du tout-venant de la production romanesque contemporaine en parlant plus souvent de la conduction divine qui régirait constamment le sort de ses personnages; ces personnages, de leur côté, s'en "remett(ent)» tout aussi constamment «à Dieu du soin de disposer de (leur) sort selon sa volonté» (t. I, p. I79). Tout cela, qu’on ne retrouve donc pas ailleurs, ne cherche pourtant pas à nous faire accéder à quelque altérité orientale, qui

20. Les rares notations des Nuits au sujet de l'encadrement religieux de telles fins ne devaient de même pas dépayser leurs premiers lecteurs. Un mourant fait «sa profession de foi selon la coutume des bons musulmans à l'article de la mort» (t. II, p. 204); les rites qui la suivent semblent comporter surtout «la lecture des chapitres de l'Alcoran prescrits pour l'enterrement des morts» (t. II, p. 380), etc.

2I. Voir t. II, p. 498. 
fascinerait l'auteur et qu'il voudrait faire découvrir à son public. Ses lecteurs devaient y reconnaitre plutôt des idées et des attitudes très familières, dont les pseudo-mémoires et les nouvelles historiques plus "européens" dont ils faisaient leur pâture habituelle les entretenaient sans doute rarement mais qui n'en faisaient pas moins la trame d'un Ancien Régime dont on n'imaginait seulement pas qu'il pût jamais s'effacer.

Les Nuits sont contemporaines de ce que nous appelons, depuis Paul Hazard, la crise de conscience européenne. Elles invitent peut-être surtout à ne pas surestimer l'ampleur de cette "crise", que nous sommes portés comme d'instinct à majorer parce que notre modernité tout entière en découle largement. Il conviendrait donc de ne pas trop oublier qu'il ne devait ni ne pouvait s'agir à l'époque que de commencements premiers fort circonscrits, autour desquels de très vieilles psycho-logiques, que les sujets de Louis XIV pouvaient partager toujours avec ceux de Haroun-al Raschid, restaient largement intactes. Et qu'avant de se prêter, des Lettres persanes à Zadig et au-delà, aux usages philosophiques qu'on sait, la fiction orientale aura d'abord proposé, sous la plume heureuse d'Antoine Galland, une manière de doublure souriante du monde habituel de ses lecteurs. L'Ancien Régime y aura réussi pour une fois — qui, bien sûr, ne faisait pas coutume - à fraterniser avec l'islam. 